\title{
KURZWEIL INTEGRAL FOR RIESZ SPACE-VALUED FUNCTIONS: UNIFORM CONVERGENCE THEOREM
}

\author{
G. A. Monteiro* - R. Fernandez** \\ (Communicated by David Buhagiar)
}

\begin{abstract}
Our objective here is to prove that the uniform convergence of a sequence of Kurzweil integrable functions implies the convergence of the sequence formed by its corresponding integrals.
\end{abstract}

(c) 2012

Mathematical Institute

Slovak Academy of Sciences

\section{Introduction}

The Kurzweil integral for Riesz space-valued functions has been investigated in various papers (see, for example, [2], [3], [5], [7], [9]). In [9], it was proved that:

If $X$ is a Dedekind $\sigma$-complete weakly $\sigma$-distributive Riesz space, $f \in X^{[a, b]}$ is a bounded function and $\left(f_{n}\right)_{n \in \mathbb{N}}$ is a sequence of Kurzweil integrable functions on $[a, b]$ such that $f_{n} \stackrel{u}{\rightarrow} f$, then

$$
f \text { is Kurzweil integrable on }[a, b] \text { and } \int_{a}^{b} f_{n} \stackrel{o}{\longrightarrow} \int_{a}^{b} f \text {. }
$$

Our purpose here is to obtain (1) without the assumption that $f$ is bounded (see Theorem 3.3 in the sequel).

2010 Mathematics Subject Classification: Primary 28B15.

Keywords: Kurzweil integral, uniform convergence, (D)-sequences. 


\section{G. A. MONTEIRO — R. FERNANDEZ}

\section{Notations and definitions}

A Riesz space $X$ is a real vector space on which a partial ordering $\leq$ compatible with its vector space structure is defined, that is, $(X, \leq)$ is a lattice. In what follows, $X$ denotes a Riesz space.

A sequence $\left(x_{n}\right)_{n \in \mathbb{N}}$ of elements of $X$ is said to be $(o)$-convergent to $x \in X$, and we write $x_{n} \stackrel{o}{\rightarrow} x$, if there exists a nonincreasing sequence $\left(a_{n}\right)_{n \in \mathbb{N}}$ of elements of $X$ such that

$$
a_{n} \searrow 0 \text { and }\left|x_{k}-x\right| \leq a_{k}, \quad \text { for all } k \in \mathbb{N} .
$$

It is possible to prove that $x_{n} \stackrel{o}{\rightarrow} x$ if and only if $\left(x_{n}\right)_{n \in \mathbb{N}}$ is bounded and

$$
x=\bigvee_{m=1}^{\infty}\left(\bigwedge_{i=m}^{\infty} x_{i}\right)=\bigwedge_{m=1}^{\infty}\left(\bigvee_{i=m}^{\infty} x_{i}\right)
$$

This charactherization of order convergence, whose proof can be found in [4], will be useful later.

A sequence $\left(f_{n}\right)_{n \in \mathbb{N}}$ of elements of $X^{[a, b]}$ is said to be uniformly convergent to $f \in X^{[a, b]}$, and we write $f_{n} \stackrel{u}{\rightarrow} f$, if there exists a nonincreasing sequence $\left(a_{n}\right)_{n \in \mathbb{N}}$ of elements of $X$ such that

$a_{n} \searrow 0$ and $\left|f_{k}(x)-f(x)\right| \leq a_{k}, \quad$ for all $\quad x \in[a, b] \quad$ and $\quad k \in \mathbb{N}$.

It is easy to check that a sequence $\left(f_{n}\right)_{n \in \mathbb{N}}$ of elements of $X^{[a, b]}$ which is uniformly convergent is also Cauchy uniformly, that is, there exists a nonincreasing sequence $\left(b_{n}\right)_{n \in \mathbb{N}}$ of elements of $X$ such that $b_{n} \searrow 0$ and, for each $k \in \mathbb{N}$,

$$
\left|f_{i}(x)-f_{j}(x)\right| \leq b_{k}, \quad \text { for all } \quad x \in[a, b] \text { and } i, j \geq k \text {. }
$$

A bounded double sequence $\left(a_{i j}\right)_{i j}$ of elements of $X$ is said to be a $(D)$-sequence if, for each $i \in \mathbb{N}$, the sequence $\left(a_{i j}\right)_{j \in \mathbb{N}}$ is nonincreasing and $a_{i j} \searrow 0$ as $j \rightarrow \infty$. These double sequences, as Riečan has mentioned in [7], can be used to indicate an idea of proximity.

The Riesz space $X$ is said to be a Dedekind $\sigma$-complete weakly $\sigma$-distributive Riesz space, if it is Dedekind $\sigma$-complete Riesz space (i.e., every non-empty, countable subset that has upper bound admits a least upper bound or supremum) and

$$
\bigwedge_{\varphi \in \mathbb{N}^{\mathbb{N}}}\left(\bigvee_{i=1}^{\infty} a_{i \varphi(i)}\right)=0, \quad \text { for all }(D) \text {-sequence }\left(a_{i j}\right)_{i j} \text { of elements of } X
$$


A function $f \in X^{[a, b]}$, where $X$ is a Dedekind $\sigma$-complete weakly $\sigma$-distributive Riesz space, is said to be Kurzweil integrable on $[a, b]$ if there exists $L \in X$, satisfying:

there is a $(D)$-sequence $\left(a_{i j}\right)_{i j}$ of elements of $X$ such that, for every $\varphi \in \mathbb{N}^{\mathbb{N}}$, there exists $\delta=\delta(\varphi) \in \mathbb{R}_{+}^{[a, b]}$ such that

$$
\left|\sum_{\Pi} f-L\right| \leq \bigvee_{i=1}^{\infty} a_{i \varphi(i)}, \quad \text { for all } \quad \Pi \in \mathcal{A}_{[a, b]}(\delta) .
$$

The set $\mathcal{A}_{[a, b]}(\delta)$ consists of all partitions $\Pi$ of $[a, b], \Pi:=\left\{\left(I_{k}, \alpha_{k}\right)\right\}_{k \in \Lambda}, \Lambda \subset \mathbb{N}$ finite and $\left.\alpha_{k} \in I_{k} \subset\right] \alpha_{k}-\delta\left(\alpha_{k}\right), \alpha_{k}+\delta\left(\alpha_{k}\right)\left[\right.$, for all $k \in \Lambda$. The element $\sum_{\Pi} f$ denotes the Riemann sum of $f$ with respect to $\Pi$, that is, $\sum_{k \in \Lambda} f\left(\alpha_{k}\right) m\left(I_{k}\right)$, where $m$ is the Lebesgue measure in $\mathbb{R}$.

The element $L$ that satisfies (2) is unique, it is called the Kurzweil integral of $f$ on $[a, b]$ and we denote by $\int_{a}^{b} f$.

The linear properties for the Kurzweil integral in this sense can be easily established (see [7, Theorem 4]).

\section{Uniform Convergence theorem}

The proof of Theorem 3.3 follows closely the ideas presented by Riečan and Vrábelová in [9]. For this, we need the following lemma whose proof can be found in [6, Lema 3.2.2] or [8].

Lemma 3.1. Let $X$ be a Dedekind $\sigma$-complete weakly $\sigma$-distributive Riesz space. If $\left(a_{n i j}\right)_{n i j}$ is a triple sequence of elements of $X$ such that, for each $n \in \mathbb{N}$, $\left(a_{n i j}\right)_{i j}$ is a $(D)$-sequence, then there is a double sequence $\left(b_{i j}\right)_{i j}$ of elements of $X$ such that, for all $S \in X^{+} \backslash\{0\}$ and $\varphi \in \mathbb{N}^{\mathbb{N}}$,

$$
S \wedge\left(\sum_{n=1}^{k} \bigvee_{i=1}^{\infty} a_{n i \varphi(i+n)}\right) \leq \bigvee_{j=1}^{\infty}\left(S \wedge b_{j \varphi(j)}\right), \quad \text { for all } k \in \mathbb{N}
$$

Moreover, $\left(S \wedge b_{i j}\right)_{i j}$ is a $(D)$-sequence for all $S \in X^{+} \backslash\{0\}$. 


\section{G. A. MONTEIRO — R. FERNANDEZ}

In order to prove the Uniform Convergence theorem we will also use the next result, concerning convergence, presented in [6].

Proposition 3.2. Let $X$ be a Dedekind $\sigma$-complete weakly $\sigma$-distributive Riesz space and $\left(f_{n}\right)_{n \in \mathbb{N}}$ be a sequence of Kurzweil integrable functions on $[a, b]$. If $\left(f_{n}\right)_{n \in \mathbb{N}}$ is Cauchy uniformly, then there exists $\bigwedge_{m=1}^{\infty}\left(\bigvee_{i=m}^{\infty} \int_{a}^{b} f_{i}\right)$ and

$$
\int_{a}^{b} f_{n} \stackrel{o}{\longrightarrow} \bigwedge_{m=1}^{\infty}\left(\bigvee_{i=m}^{\infty} \int_{a}^{b} f_{i}\right)
$$

Pr o o f. It is sufficient to show that the sequence $\left(\int_{a}^{b} f_{n}\right)_{n \in \mathbb{N}}$ is bounded and

$$
\bigwedge_{m=1}^{\infty}\left(\bigvee_{i=m}^{\infty} \int_{a}^{b} f_{i}\right) \leq \bigvee_{m=1}^{\infty}\left(\bigwedge_{i=m}^{\infty} \int_{a}^{b} f_{i}\right)
$$

considering that the other inequality always holds for bounded sequences.

Since $\left(f_{n}\right)_{n \in \mathbb{N}}$ is Cauchy uniformly, there exists a nonincreasing sequence $\left(b_{n}\right)_{n \in \mathbb{N}}$ of elements of $X$ such that $b_{n} \searrow 0$ and, for each $k \in \mathbb{N}$,

$$
\left|f_{i}(x)-f_{j}(x)\right| \leq b_{k}, \quad \text { for all } \quad x \in[a, b] \text { and } i, j \geq k .
$$

Given $n \in \mathbb{N}$, for $i, j \geq n$, we obtain $f_{i}(x) \leq f_{j}(x)+b_{n}, x \in[a, b]$, and then

$$
\int_{a}^{b} f_{i} \leq\left(\int_{a}^{b} f_{j}\right)+b_{n}(b-a), \quad \text { for all } i, j \geq n .
$$

In particular, it follows that,

$$
\left(\int_{a}^{b} f_{1}\right)-b_{1}(b-a) \leq \int_{a}^{b} f_{k} \leq\left(\int_{a}^{b} f_{1}\right)+b_{1}(b-a), \quad k \in \mathbb{N}
$$

what proves that $\left(\int_{a}^{b} f_{n}\right)_{n \in \mathbb{N}}$ is bounded.

By (4), for all $n \in \mathbb{N}$, we have

$$
\left(\bigvee_{i=n}^{\infty} \int_{a}^{b} f_{i}\right) \leq\left(\bigwedge_{j=n}^{\infty} \int_{a}^{b} f_{j}\right)+b_{n}(b-a)
$$


which implies that,

$$
\bigwedge_{m=1}^{\infty}\left(\bigvee_{k=m}^{\infty} \int_{a}^{b} f_{k}\right)-\bigvee_{m=1}^{\infty}\left(\bigwedge_{k=m}^{\infty} \int_{a}^{b} f_{k}\right) \leq\left(\bigvee_{i=n}^{\infty} \int_{a}^{b} f_{i}\right)-\left(\bigwedge_{j=n}^{\infty} \int_{a}^{b} f_{j}\right) \leq b_{n}(b-a) .
$$

Noting that $\left(b_{n}(b-a)\right) \searrow 0$, as $n \rightarrow \infty$, the inequality (3) is proved.

Theorem 3.3 (Uniform Convergence theorem). Let $X$ be a Dedekind $\sigma$-complete weakly $\sigma$-distributive Riesz space and $\left(f_{n}\right)_{n \in \mathbb{N}}$ be a sequence of Kurzweil integrable functions on $[a, b]$. If $f_{n} \stackrel{u}{\rightarrow} f$, then $f$ is Kurzweil integrable on $[a, b]$ and

$$
\int_{a}^{b} f_{n} \stackrel{o}{\longrightarrow} \int_{a}^{b} f
$$

P r o of. Since $f_{n} \stackrel{u}{\rightarrow} f$, there exists a nonincreasing sequence $\left(u_{n}\right)_{n \in \mathbb{N}}$ of elements of $X$ such that

$$
u_{n} \searrow 0 \text { and }\left|f_{k}(x)-f(x)\right| \leq u_{k}, \quad \text { for all } \quad x \in[a, b] \text { and } k \in \mathbb{N} \text {. }
$$

By $(5),\left(f_{n}\right)_{n \in \mathbb{N}}$ is Cauchy uniformly and, therefore, by Proposition 3.2, there exists $L \in X$ such that $\int_{a}^{b} f_{n} \stackrel{o}{\rightarrow} L$. Thus, there exists a nonincreasing sequence $\left(w_{n}\right)_{n \in \mathbb{N}}$ of elements of $\stackrel{a}{X}$ such that

$$
w_{n} \searrow 0 \text { and }\left|\int_{a}^{b} f_{k}-L\right| \leq w_{k}, \quad \text { for all } \quad k \in \mathbb{N} .
$$

Since $f_{n}$ is Kurzweil integrable on $[a, b]$, for each $n \in \mathbb{N}$, there is a $(D)$-sequence $\left(a_{i j}^{(n)}\right)_{i j}$ of elements of $X$ such that, for every $\psi \in \mathbb{N}^{\mathbb{N}}$, there exists $\delta_{n}=\delta_{n}(\psi) \in \mathbb{R}_{+}^{[a, b]}$ such that

$$
\left|\sum_{\Pi} f_{n}-\int_{a}^{b} f_{n}\right| \leq \bigvee_{i=1}^{\infty} a_{i \psi(i)}^{(n)}, \quad \text { for all } \quad \Pi \in \mathcal{A}_{[a, b]}\left(\delta_{n}\right) .
$$

Using (5), (6) and (7), we have

$$
\begin{aligned}
\left|\sum_{\Pi} f-L\right| & \leq\left|\sum_{\Pi} f-\sum_{\Pi} f_{n}\right|+\left|\sum_{\Pi} f_{n}-\int_{a}^{b} f_{n}\right|+\left|\int_{a}^{b} f_{n}-L\right| \\
& \leq\left|\sum_{k \in \Lambda}\left(f\left(\alpha_{k}\right)-f_{n}\left(\alpha_{k}\right)\right) m\left(I_{k}\right)\right|+\bigvee_{i=1}^{\infty} a_{i \psi(i)}^{(n)}+w_{n}
\end{aligned}
$$




\section{G. A. MONTEIRO - R. FERNANDEZ}

$$
\begin{aligned}
& \leq\left(\sum_{k \in \Lambda}\left|f\left(\alpha_{k}\right)-f_{n}\left(\alpha_{k}\right)\right| m\left(I_{k}\right)\right)+\bigvee_{i=1}^{\infty} a_{i \psi(i)}^{(n)}+w_{n} \\
& \leq\left(\sum_{k \in \Lambda} u_{n} m\left(I_{k}\right)\right)+\bigvee_{i=1}^{\infty} a_{i \psi(i)}^{(n)}+w_{n} \\
& =u_{n}(b-a)+\bigvee_{i=1}^{\infty} a_{i \psi(i)}^{(n)}+w_{n},
\end{aligned}
$$

that is,

$$
\left|\sum_{\Pi} f-L\right| \leq u_{n}(b-a)+w_{n}+\bigvee_{i=1}^{\infty} a_{i \psi(i)}^{(n)}, \quad \text { for all } \quad \Pi \in \mathcal{A}_{[a, b]}\left(\delta_{n}\right) .
$$

Consider a triple sequence $\left(c_{n i j}\right)_{n i j}$ of elements of $X$ defined by

$$
c_{n i j}:=\left\{\begin{array}{ll}
w_{j}+u_{j}(b-a) & \text { if } \quad n=1 \\
a_{i j}^{(n-1)} & \text { if } \quad n \geq 2
\end{array}, \quad i, j \in \mathbb{N} .\right.
$$

Note that, for every fixed $n \in \mathbb{N}$, the bounded double sequence $\left(c_{n i j}\right)_{i j}$ is a $(D)$-sequence of elements of $X$ because, for each $i \in \mathbb{N},\left(c_{n i j}\right)_{j \in \mathbb{N}}$ is a nonincreasing sequence and $c_{n i j} \searrow 0$, as $j \rightarrow \infty$.

Let $S:=u_{1}(b-a)+w_{1}+\bigvee_{i, j=1}^{\infty} a_{i j}^{(1)}$. If $S=0$, then $u_{1}=0$ and, by $(5)$, we obtain $f \equiv f_{k}$, for all $k \in \mathbb{N}$. Assuming $S \in X^{+} \backslash\{0\}$, there exists, by Lemma 3.1, a double sequence $\left(b_{i j}\right)_{i j}$ of elements of $X$ such that $\left(S \wedge b_{i j}\right)_{i j}$ is a $(D)$-sequence and, for every $\varphi \in \mathbb{N}^{\mathbb{N}}$,

$$
S \wedge\left(\sum_{r=1}^{n} \bigvee_{i=1}^{\infty} c_{r i \varphi(i+r)}\right) \leq \bigvee_{j=1}^{\infty}\left(S \wedge b_{j \varphi(j)}\right), \quad \text { for all } \quad n \in \mathbb{N}
$$

Now we will prove that the $(D)$-sequence $\left(a_{i j}\right)_{i j}:=\left(S \wedge b_{i j}\right)_{i j}$ satisfies the Kurzweil integrability condition for $f$.

Given $\varphi \in \mathbb{N}^{\mathbb{N}}$, consider $p:=\min _{i \in \mathbb{N}} \varphi(i+1)$ and let $\psi \in \mathbb{N}^{\mathbb{N}}$ be the function defined by $\psi(j)=\varphi(j+p+1)$, for $j \in \mathbb{N}$. By $(8)$, for $\Pi \in \mathcal{A}_{[a, b]}\left(\delta_{p}\right), \delta_{p}=\delta_{p}(\psi)$, we obtain

$$
\left|\sum_{\Pi} f-L\right| \leq u_{p}(b-a)+w_{p}+\bigvee_{i=1}^{\infty} a_{i \varphi(i+p+1)}^{(p)} .
$$




\section{KURZWEIL INTEGRAL FOR RIESZ SPACE-VALUED FUNCTIONS}

Note that $\bigvee_{j=1}^{\infty}\left(u_{\varphi(j+1)}(b-a)+w_{\varphi(j+1)}\right)=u_{p}(b-a)+w_{p}$. Thus we can write

$$
\left|\sum_{\Pi} f-L\right| \leq \bigvee_{j=1}^{\infty}\left(u_{\varphi(j+1)}(b-a)+w_{\varphi(j+1)}\right)+\bigvee_{i=1}^{\infty} a_{i \varphi(i+p+1)}^{(p)},
$$

that is,

$$
\left|\sum_{\Pi} f-L\right| \leq \bigvee_{j=1}^{\infty} c_{1 j \varphi(j+1)}+\bigvee_{i=1}^{\infty} c_{(p+1) i \varphi(i+p+1)} \leq \sum_{r=1}^{p+1} \bigvee_{i=1}^{\infty} c_{r i \varphi(i+r)} .
$$

On the other hand, by (8), for $n=1$, we have

$$
\left|\sum_{\Pi} f-L\right| \leq u_{1}(b-a)+w_{1}+\bigvee_{i, j=1}^{\infty} a_{i j}^{(1)}=S,
$$

whenever $\Pi \in \mathcal{A}_{[a, b]}\left(\delta_{1}\right)$, where $\delta_{1} \in \mathbb{R}_{+}^{[a, b]}$.

Assuming that $\delta:=\min \left\{\delta_{1} ; \delta_{p}\right\}$, for $\Pi \in \mathcal{A}_{[a, b]}(\delta)$ we have, by (9), (10) and (11),

$$
\left|\sum_{\Pi} f-L\right| \leq S \wedge\left(\sum_{r=1}^{p+1} \bigvee_{i=1}^{\infty} c_{r i \varphi(i+r)}\right) \leq \bigvee_{j=1}^{\infty}\left(S \wedge b_{j \varphi(j)}\right)=\bigvee_{j=1}^{\infty} a_{j \varphi(j)}
$$

Thus $f$ is Kurzweil integrable on $[a, b]$, and moreover $\int_{a}^{b} f_{n} \stackrel{o}{\rightarrow} L=\int_{a}^{b} f$.

\section{REFERENCES}

[1] BARTLE, R.: A Modern Theory of Integration. Grad. Stud. Math., Amer. Math. Soc., Providence, RI, 2001.

[2] BOCCUTO, A.: Differential and integral calculus in Riesz space, Tatra Mt. Math. Publ. 14 (1998), 293-323.

[3] BOCCUTO, A.-SKVORTSOV, V. A.: Henstock-Kurzweil type integration of Riesz-spacevalued functions and applications to Walsh series, Real Anal. Exchange 29 (2003-04), 419-438.

[4] LuXemburG, W. A.-ZAAnen, A. C.: Riesz spaces I, North-Holland, Amsterdam, 1971.

[5] McGILL, P.: Integration in vector lattices, J. London Math. Soc. (2) 11 (1975), 347-360.

[6] MONTEIRO, G. A.: Integral de Kurzweil para funções a valores em um espaço de Riesz - uma introdução. Master Dissertation, Universidade de São Paulo, São Paulo, 2007.

[7] RIEČAN, B.: On the Kurzweil integral for functions with values in ordered spaces I, Acta Math. Univ. Comenian. (N.S.) 56-57 (1990), 75-83. 


\section{G. A. MONTEIRO - R. FERNANDEZ}

[8] RIEČAN, B.-VOLAUF, P.: On a technical lemma in lattice ordered groups, Acta Math. Univ. Comenian. (N.S.) 44-45 (1984), 31-35.

[9] RIEČAN, B.-VRÁBELOVÁ, M.: On the Kurzweil integral for functions with values in ordered spaces II, Math. Slovaca 43 (1993), 471-475.

Received 5. 7. 2009

Accepted 5. 2. 2010

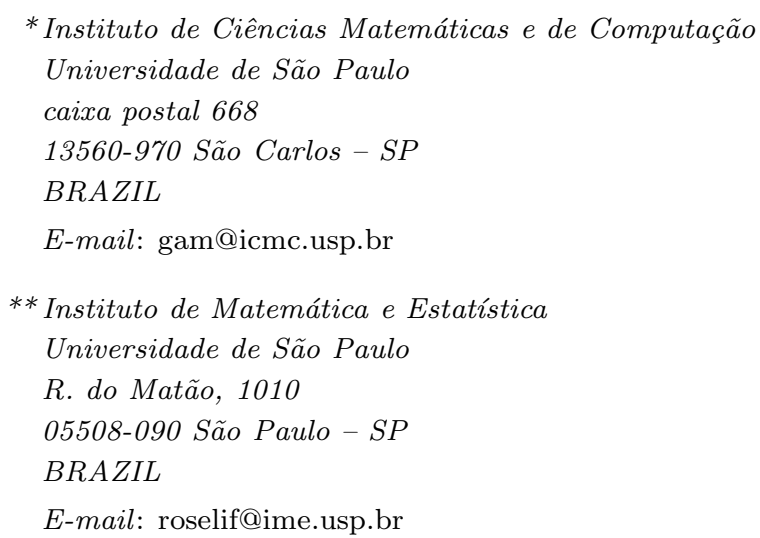

